Supporting Information

\title{
Hitchhiking Nanoparticles: Mesenchymal Stem Cell-Mediated Delivery of Theranostic Nanoparticles
}

Dominyka Dapkute ${ }^{1,2} *$, Marijus Pleckaitis ${ }^{1,2}$, Danute Bulotiene ${ }^{1}$, Dainius Daunoravicius ${ }^{3}$,

Ricardas Rotomskis ${ }^{1,4}$, Vitalijus Karabanovas ${ }^{1,5 *}$

${ }^{1}$ Biomedical Physics Laboratory, National Cancer Institute, P. Baublio 3B, 08406 Vilnius,

Lithuania

${ }^{2}$ Life Sciences Center, Vilnius University, Sauletekio ave. 7, 10223 Vilnius, Lithuania

${ }^{3}$ Department of Pathology, Forensic Medicine and Pharmacology, Faculty of Medicine, Vilnius

University, M. K. Ciurlionio 21/27, 03101 Vilnius, Lithuania

${ }^{4}$ Biophotonics group, Laser Research Centre, Vilnius University, Sauletekio ave. 10, 10223

Vilnius, Lithuania

${ }^{5}$ Department of Chemistry and Bioengineering, Vilnius Gediminas Technical University,

Sauletekio ave. 11, 10221 Vilnius, Lithuania 
A

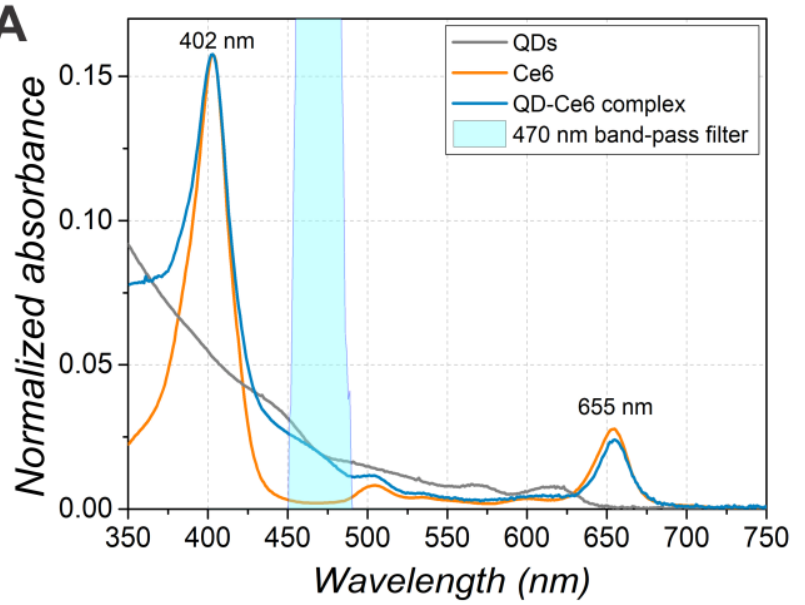

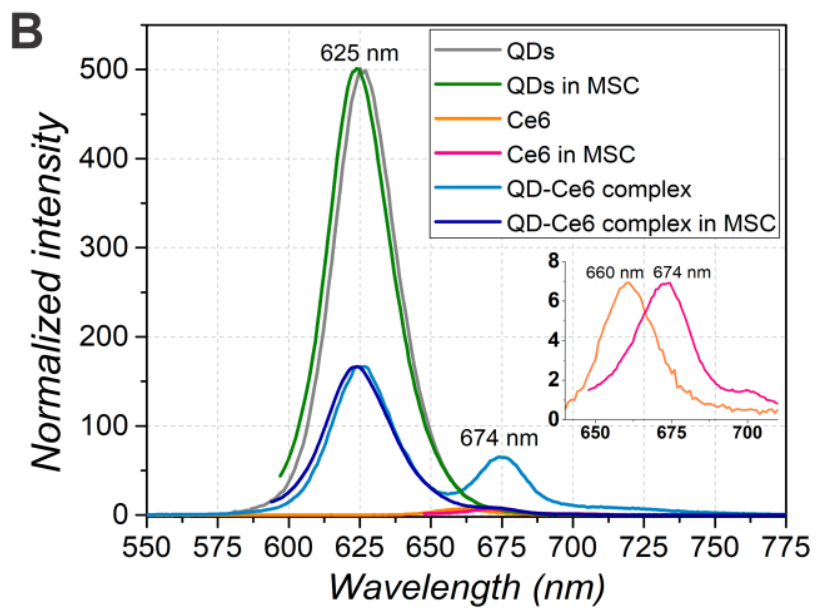

Figure S1. QD, Ce6, and QD-Ce6 complex absorption and photoluminescence spectra. (A) Normalized absorption spectra of QDs, Ce6, and QD-Ce6 complex in PBS. Light blue spectrum indicates transmission of the $470 \mathrm{~nm}$ filter in MAX-302 xenon light source used for the irradiation of QD-Ce6 complex throughout the study. (B) Comparison of QDs, Ce6, and QD-Ce6 complex fluorescence spectral measurements in PBS and in MSCs. Inset shows the enlarged area at the Ce6 fluorescence region. 

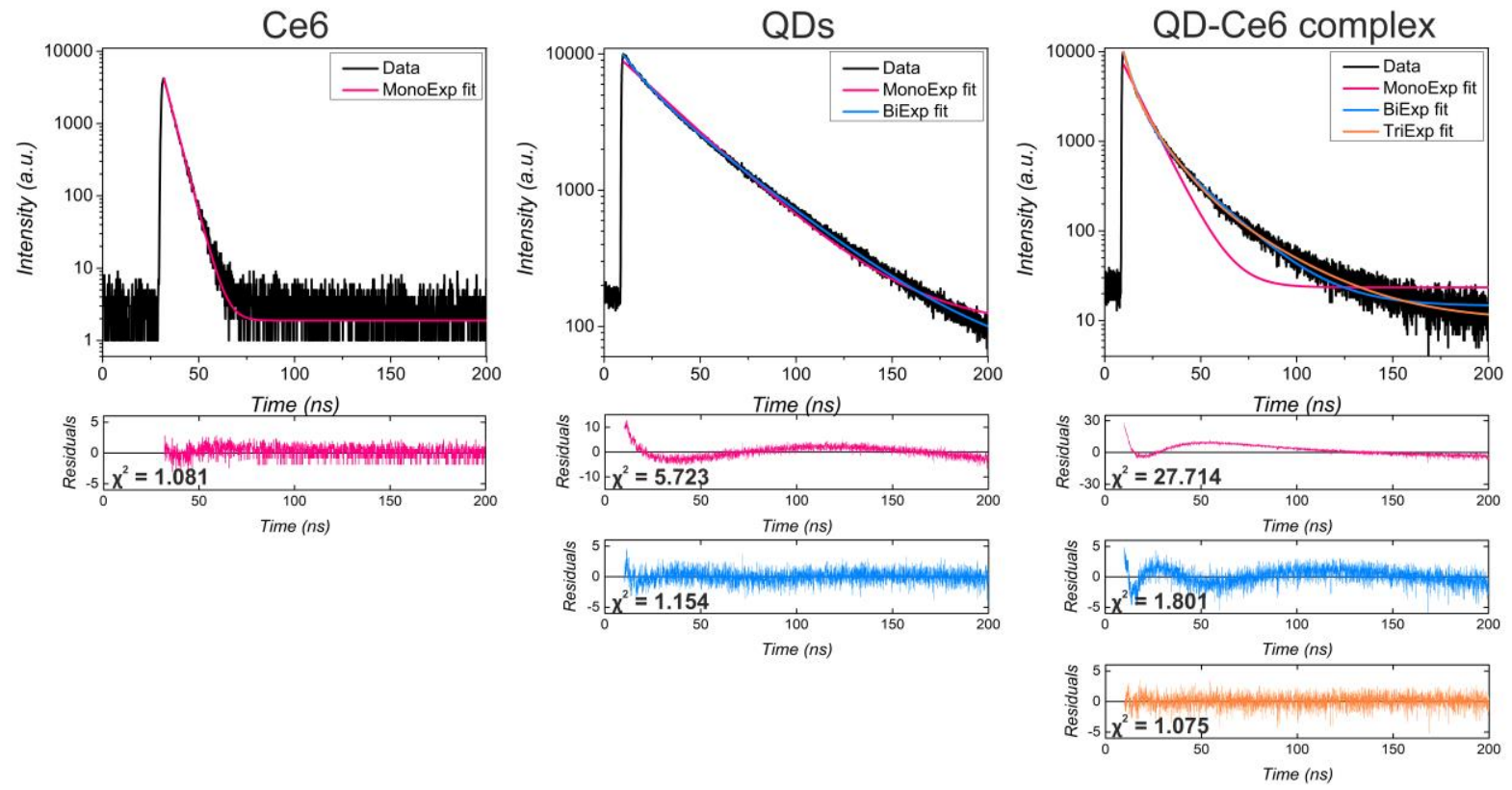

Figure S2. Fluorescence decay curves and residuals of QD, Ce6, and QD-Ce6 complex. Fluorescence decay curves of Ce6, QDs, and QD-Ce6 complex, fitted with various exponential functions and their residuals of respective fitting (mono-exponential fit represented in magenta, bi-exponential - blue, and tri-exponential - orange). $\chi^{2}$ values indicate optimal exponential fit. 

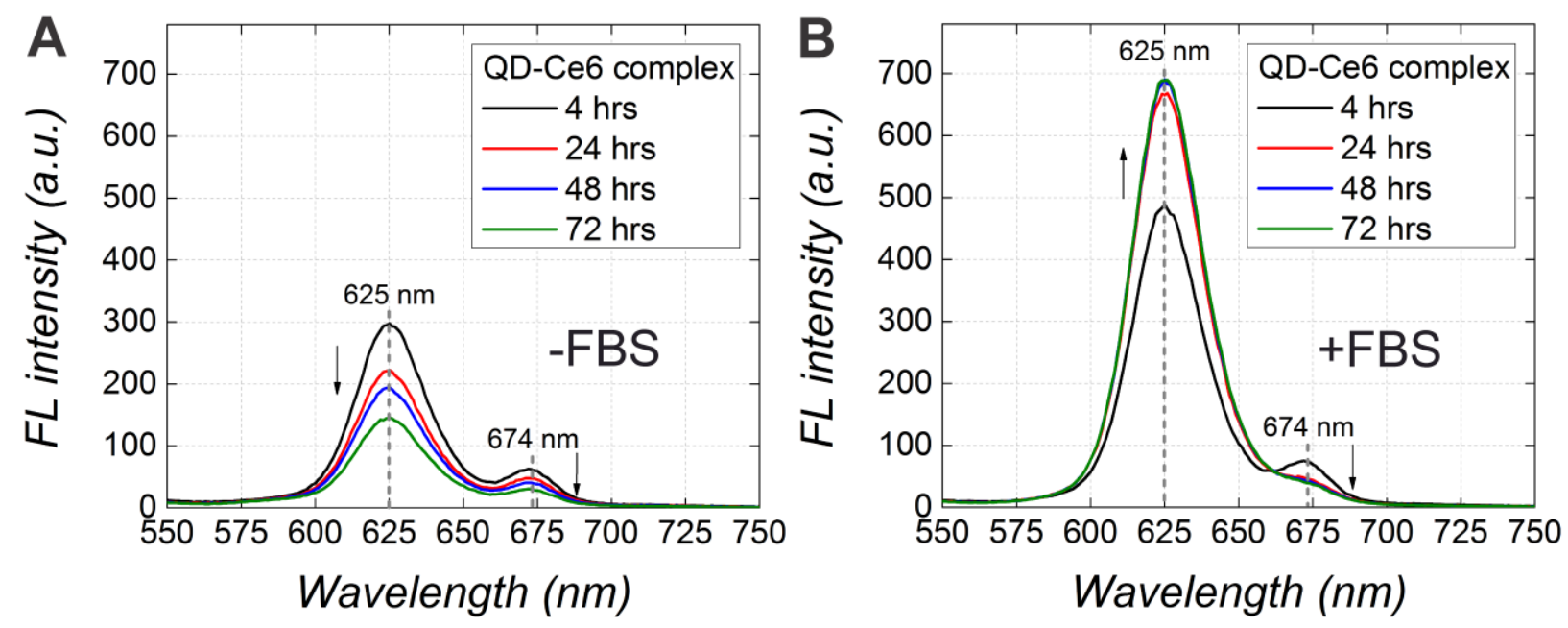

Figure S3. Unnormalized (raw) QD-Ce6 complex photoluminescence spectra in different media. (A) QD-Ce6 complex (ratio 1:100) FL spectra over time in serum-free (-FBS) medium $\left(\lambda_{\mathrm{ex}}=470\right.$ nm). (B) QD-Ce6 complex (ratio 1:100) FL spectra over time in serum-supplemented (+FBS) medium $\left(\lambda_{\mathrm{ex}}=470 \mathrm{~nm}\right)$. Arrows indicate spectral changes. 

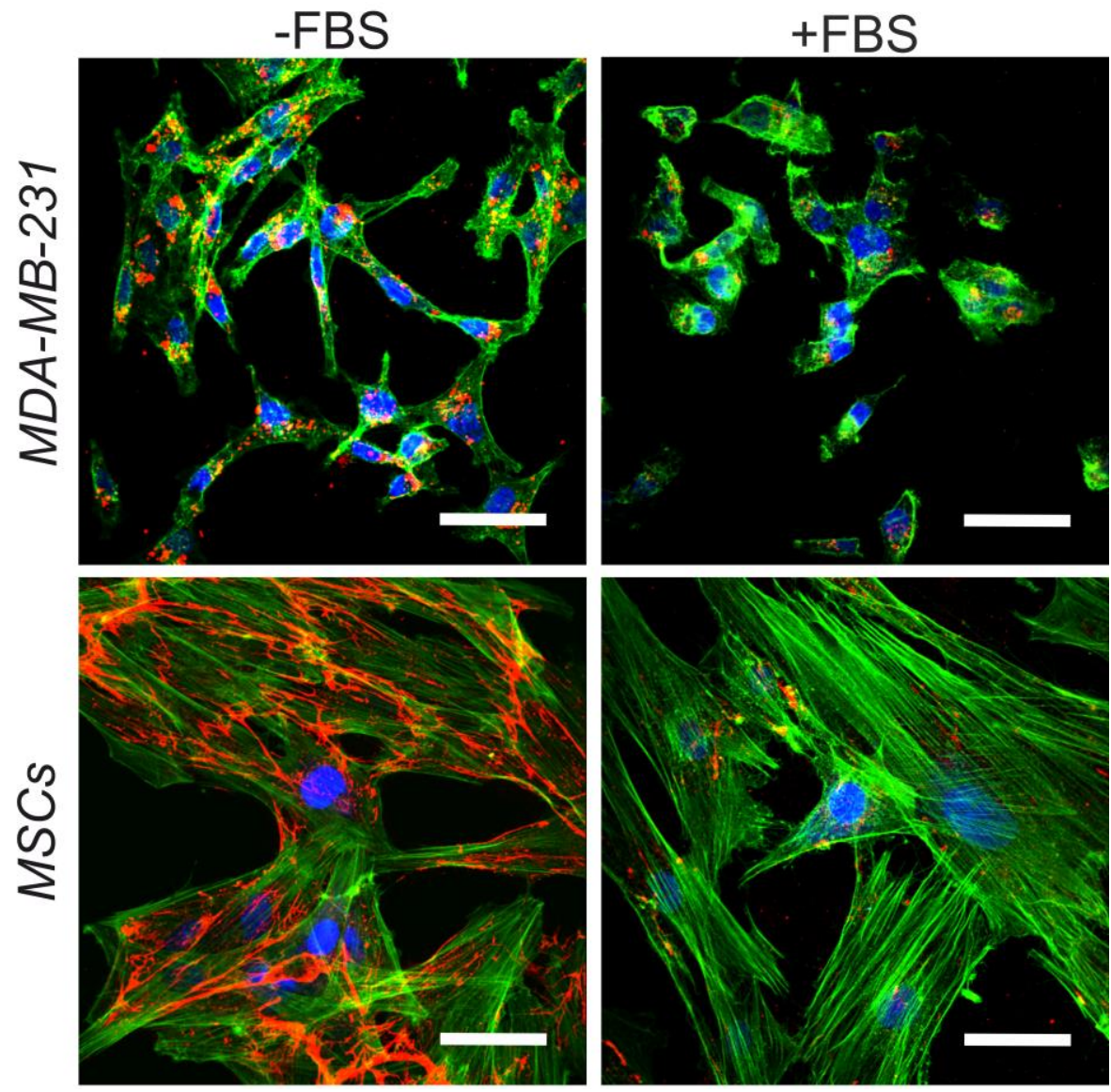

Figure S4. Confocal microscopy fluorescence images of QD-Ce6 complex accumulation in MSCs and MDA-MB-231 cancer cells in serum-free (-FBS) and serum-supplemented (+FBS) media. Green - actin, blue - nuclei, red - QD-Ce6 complex. Scale bar - $50 \mu \mathrm{m}$. 


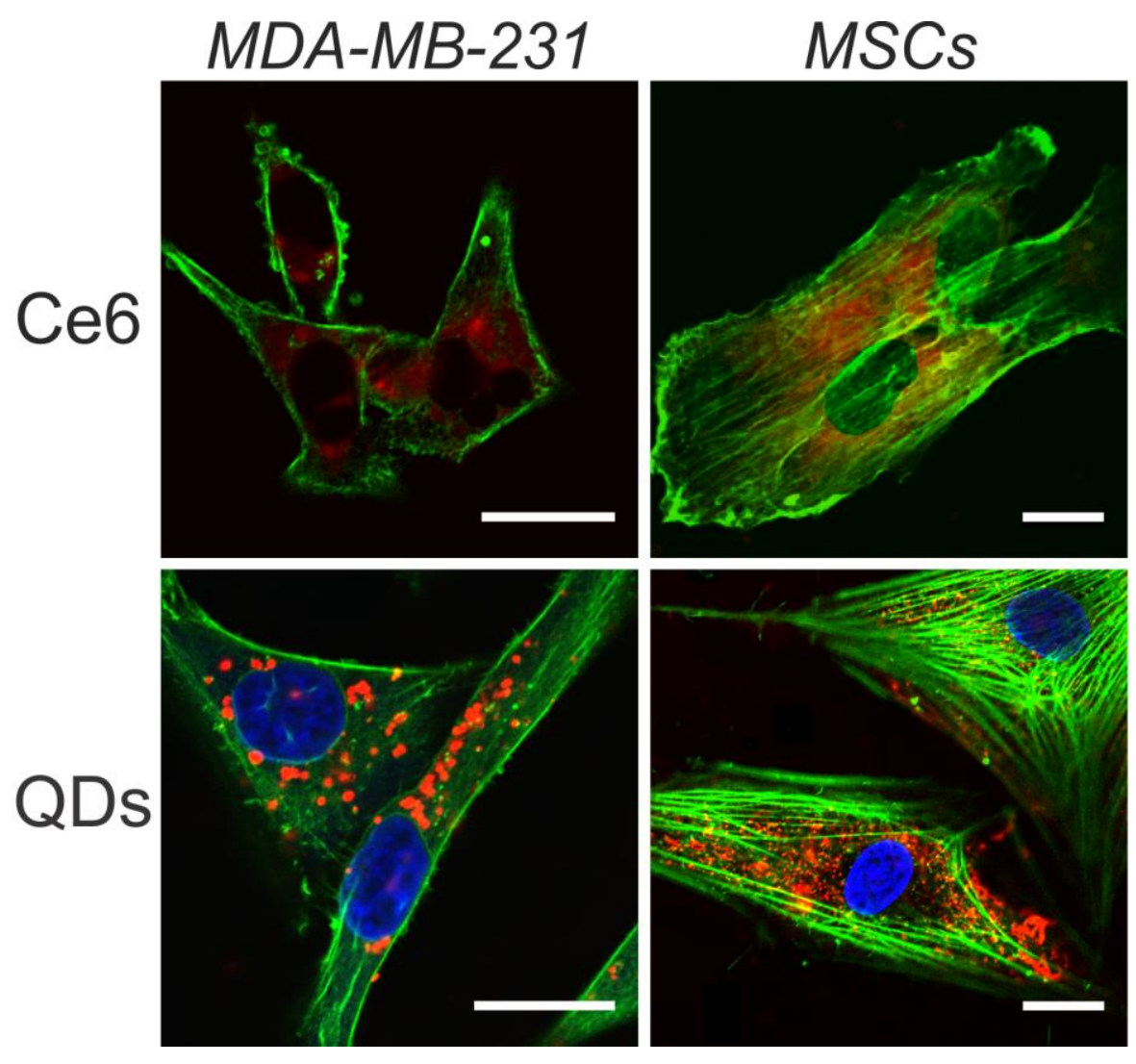

Figure S5. Subcellular localization of QDs and Ce6. Confocal microscopy fluorescence images of MDA-MB-231 cancer cells and MSCs in serum-free media showing localization of Ce6 $(1.6 \mu \mathrm{M})$ and QDs $(0.016 \mu \mathrm{M})$ after incubation for 6 hours. Green - actin, blue - nuclei, red - Ce6 or QDs. Scale bar $-20 \mu \mathrm{m}$. 


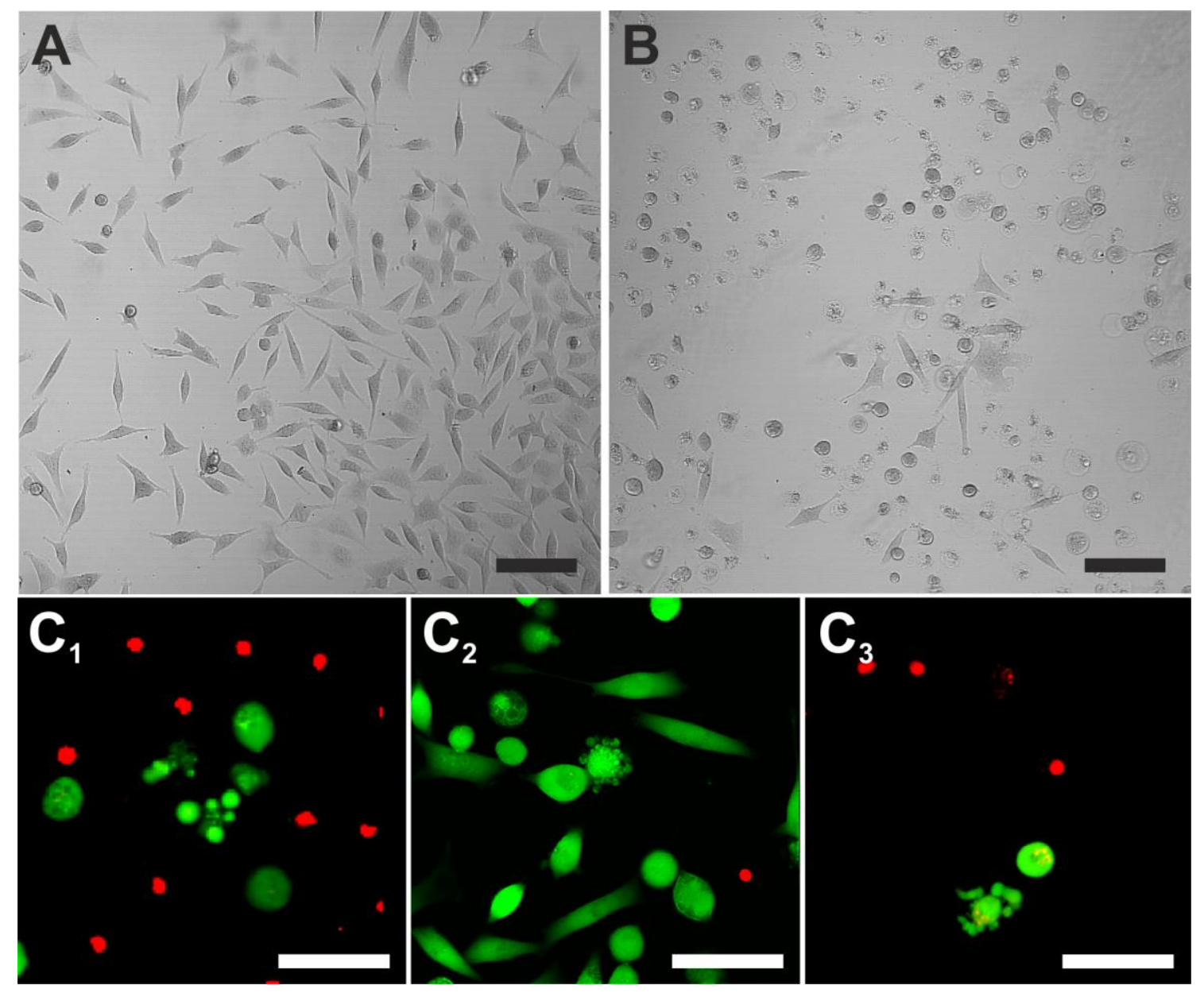

Figure S6. Apoptosis induction in MDA-MB-231 cancer cells by PDT. (A) Control MDA-MB231 cells (no irradiation). (B) MDA-MB-231 cells after incubation with QD-Ce6 complex and irradiation with $470 \mathrm{~nm}$, dose $17.7 \mathrm{~J} / \mathrm{cm}^{2}$. Scale bar - $100 \mu \mathrm{m}$. (C) Apoptotic morphology in MDAMB-231 cells after PDT. Green - calcein, red - EthD-1. Scale bar - $50 \mu \mathrm{m}$. 


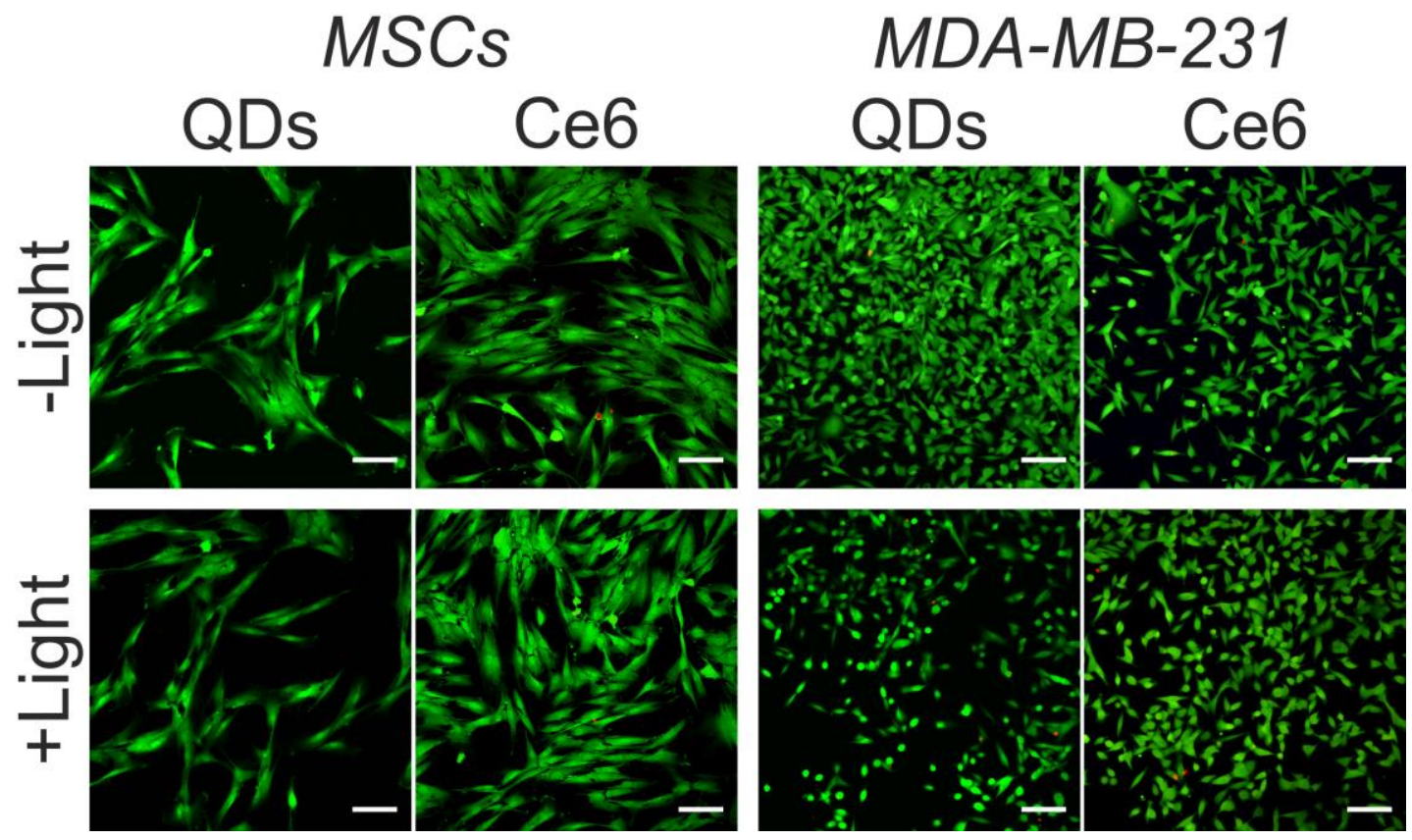

Figure S7. LIVE/DEAD labeling of MSCs and MDA-MB-231 cancer cells, incubated with QDs $(0.016 \mu \mathrm{M})$ and $\mathrm{Ce} 6(1.6 \mu \mathrm{M})$ without (-Light) and with (+Light) irradiation. Green - viable cells, red - dead cells. Irradiation with $470 \mathrm{~nm}$, dose $17.7 \mathrm{~J} / \mathrm{cm}^{2}$. Scale bar $-100 \mu \mathrm{m}$. 

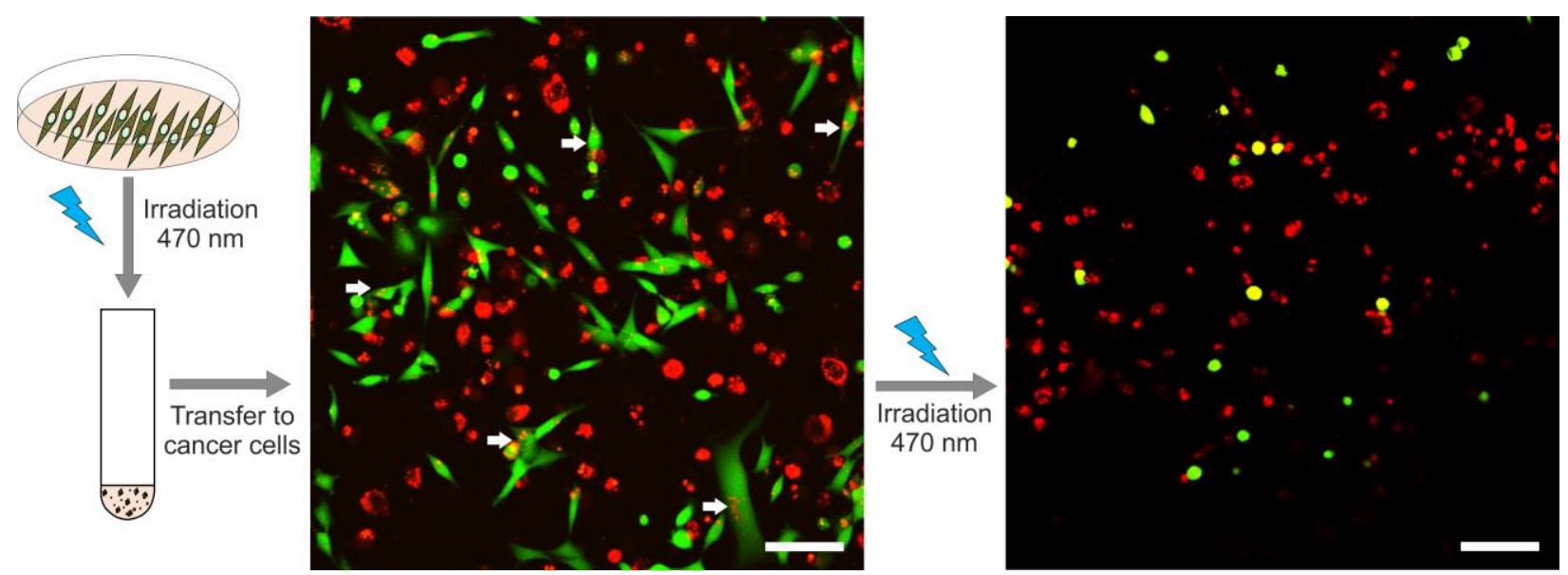

Figure S8. QD-Ce6 complex transfer from irradiated MSCs to MDA-MB-231 cancer cells. QDCe6 complex-loaded MSCs were irradiated separately and supernatant was transferred to cancer cell monoculture. For the sake of clarity, white arrows indicate some of the cancer cells with QDCe6 complex inside them. Green - viable cells (calcein), red - dead cells (EthD-1). Dead cells in the first confocal micrograph - non-viable MSCs. Scale bar $100 \mu \mathrm{m}$. 

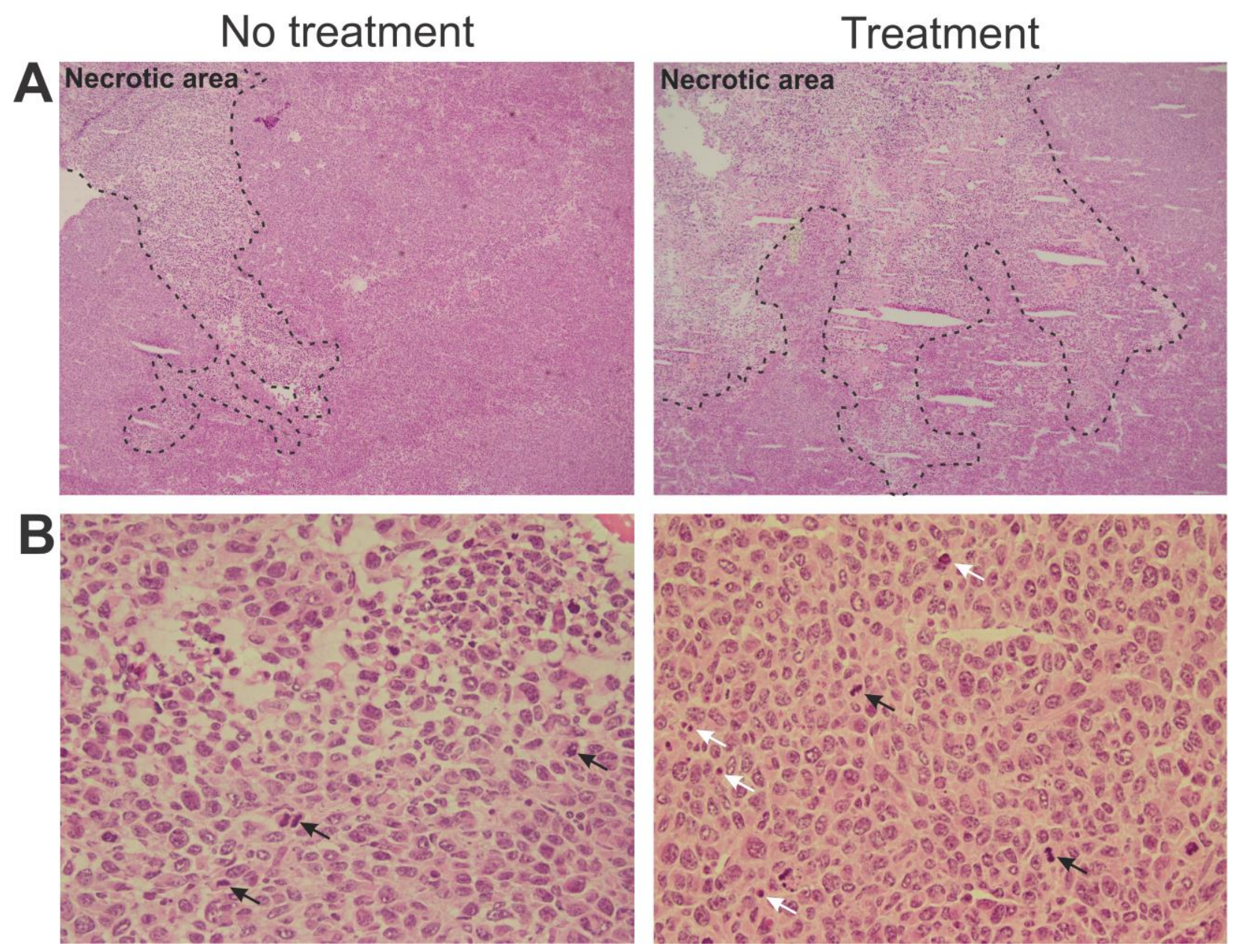

Figure S9. Microscopic examination of tumors from untreated and MSCs+QD-Ce6 complex treated mice. (A) Low magnification tumor sections with necrotic area, labeled by dashed line. HE $\times 40$. (B) Higher magnification tumor sections with mitotic (indicated by mitotic figures) and apoptotic (indicated by cell shrinkage and condensed chromosomes) cells. For the sake of clarity, a few mitotic cells are labeled by black arrows, a few apoptotic cells are labeled by white arrows. HE $\times 400$. 